\title{
EXAMPLES OF ELLIPTIC COMPLEXES
}

\author{
BY R. T. SMITH
}

Communicated by François Treves, October 16, 1975

The main purpose of this note is to give natural geometric examples of elliptic complexes for which the Poincare lemma fails. Indeed:

(a) There are natural (and even involutive) elliptic complexes which are not formally exact, and whose local cohomology is infinite (Examples 2, 3). On the other hand:

(b) An arbitrary locally exact elliptic complex need not be formally exact (cf. Example $4^{\prime}$ ).

These remarks reflect interestingly on the outstanding problem in the theory (Spencer's conjecture): Is a formally integrable formally exact elliptic complex locally exact? (See Goldschmidt [2] for a complete analysis of the formal theory.) Thus (a) demonstrates forcibly the independence of the hypotheses, whereas (b) shows that the hypothesis of formal exactness is not always necessary.

Most of our examples take the following form: Let $E$ be a subbundle of $\Lambda^{p}\left(\mathbf{R}^{n^{*}}\right)$; let $\underline{E}$ denote the sheaf of germs of sections of $E$. Then there are complexes of the following types:

$$
\begin{aligned}
& \underline{\Lambda}^{p-2} \stackrel{d}{\rightarrow} \underline{\Lambda}^{p-1} \stackrel{\pi d}{\rightarrow} \underline{\Lambda^{p}} / E ; \\
& \underline{E} \stackrel{d \mid E}{\rightarrow} \underline{\Lambda}^{p+1} \stackrel{d}{\rightarrow} \underline{\Lambda}^{p+2} .
\end{aligned}
$$

Note to begin with that the cohomology of (I) is equivalent to the space of closed sections of $E$, i.e., the solution space of a homogeneous system of equations. One of our basic observations is then:

(c) There are nontrivial examples of these types which are elliptic (cf. Examples 2, 3).

On the other hand, Spencer's conjecture itself cannot be disproved within the context of such examples: if $E$ is nontrivial, (I) is not formally exact; if (II) is elliptic (no further hypotheses), one checks it is locally exact.

\section{Constant coefficient examples.}

Example 1 (Nirenberg). An arbitrary elliptic complex need not be formally or locally exact. Over $\mathbf{C}^{n}$ construct

AMS (MOS) subject classifications (1970). Primary 35N05, 35N10; Secondary $58 \mathrm{G} 05$. Key words and phrases. Elliptic complex, formally exact complex, Dirac complex, involutive operator. 


$$
0 \rightarrow \underline{\Lambda}^{0} \oplus \underline{0} \stackrel{\bar{\partial} \oplus 0}{\longrightarrow} \underline{\Lambda}^{0,1} \oplus \underline{\Lambda}^{0} \stackrel{\overline{\partial \oplus \bar{\partial}}}{\longrightarrow} \underline{\Lambda}^{0,2} \oplus \underline{\Lambda}^{0,1} \rightarrow \cdots
$$

The cohomology at $\underline{\Lambda}^{0,1} \oplus \underline{\Lambda}^{0}$ is infinite.

We will say that a complex $\underline{E} \stackrel{D_{0}}{\longrightarrow} \underline{F} \stackrel{D_{1}}{\longrightarrow} G$ is "natural" if $D_{0}$ is induced by a surjective bundle map $\varphi_{D}: J^{k} E \rightarrow F$. This formal condition precludes artificial constructions such as the above.

EXAMPLE 2. Let $\Lambda_{ \pm}^{2}$ be the space of $*$-invariant (resp. anti-invariant) 2-forms on $\mathbf{R}^{4}$ (standard metric). Then

$$
0 \rightarrow \underline{\Lambda}^{0} \stackrel{d}{\rightarrow} \underline{\Lambda}^{1} \stackrel{\pi_{+} d}{\longrightarrow} \underline{\Lambda_{+}^{2}} \rightarrow 0
$$

is natural, elliptic, formally integrable, and involutive (cf. [3] and [4]), yet the cohomology at $\underline{\Lambda}^{1}$ is infinite. The dual complex is

$$
0 \rightarrow \underline{\Lambda}_{-}^{2} \stackrel{d \mid \Lambda^{2}}{\longrightarrow} \underline{\Lambda}^{3} \stackrel{d}{\rightarrow} \underline{\Lambda}^{4} \rightarrow 0
$$

and is locally exact as marked above. These complexes were discovered independently by Nigel Hitchin.

EXAMPLE 3. In 2 complex variables

$$
0 \rightarrow \underline{\Lambda}^{0} \stackrel{d}{\rightarrow} \underline{\Lambda}^{1} \stackrel{\pi_{1,1^{\circ d}}}{\longrightarrow} \underline{\Lambda}^{1,1} \stackrel{\partial \bar{J}}{\longrightarrow} \underline{\Lambda}^{2,2} \rightarrow 0
$$

is elliptic, but noninvolutive as reflected by the second order continuation $\partial \bar{\partial}$. The cohomology at $\underline{\Lambda}^{1}$ is again infinite, but zero otherwise. This is the dual of the well-known resolution of the sheaf of germs of pluriharmonic functions.

EXAMPLE 4. Let $\omega$ be a symplectic form on a 4-manifold $M$. Then $\wedge \omega$ : $\Lambda^{1} \rightarrow \Lambda^{3}$ is an algebraic isomorphism, and

$$
0 \rightarrow \underline{\Lambda}^{0} \stackrel{d}{\rightarrow} \underline{\Lambda}^{1} \stackrel{\pi d}{\longrightarrow} \underline{\Lambda}^{2} / \omega \stackrel{\pi d(\Lambda \omega)^{-1} d}{\longrightarrow} \underline{\Lambda}^{2} / \omega \stackrel{d}{\rightarrow} \underline{\Lambda}^{3} \stackrel{d}{\rightarrow} \underline{\Lambda}^{4} \rightarrow 0
$$

is elliptic, with local cohomology one dimensional at $\Lambda^{1}$ and exactness holding elsewhere.

One generalization of Example 2 is the following: let $F: \mathbf{R}^{k} \otimes \mathbf{R}^{n} \rightarrow \mathbf{R}^{n}$ be an orthogonal multiplication (symbol of the Dirac operator in $k$ variables). Let $E \subseteq \mathbf{R}^{n}$ be any subspace, with $E^{\perp}$ its orthocomplement. Then there is an elliptic Dirac complex

$$
0 \rightarrow \underline{E} \stackrel{D}{\rightarrow} \underline{\mathbf{R}}^{n} \stackrel{D_{1}^{*}}{\rightarrow} \underline{E}^{\perp} \rightarrow 0 .
$$

Here $\sigma_{D}$ and $\sigma_{D}$ are induced by restricting $F$ to $E$ and $E^{\perp}$ respectively. When $D$ is involutive, the exactness of a Dirac complex becomes equivalent to a combinatorial criterion, the connectedness of a certain finite graph. This uses Ehrenpreiss [1] on constant coefficient systems and Kuranishi [4] on involutive systems. Example (2) above is equivalent to the Dirac complex arising from quaternion multiplication $H \otimes H \rightarrow H$, with $E=\operatorname{Span}(1), E=\operatorname{Span}(i, j, k)$. 
Variable coefficient examples.

EXAMPLE $2^{\prime}$. There is no metric on a closed oriented manifold $M^{4}$ such that the corresponding $\Lambda_{+}^{2}$-complex is locally exact. Otherwise by sheaf theory we would find $H^{3}(M, \mathbf{R})=H^{4}(M, \mathbf{R})=0$.

EXAmpLE 3'. There are local perturbations of (3) such that the cohomology at $\Lambda^{\mathbf{1}}$ is finite. This is equivalent to exhibiting perturbations of the homogeneous Cauchy-Riemann equations for holomorphic functions with finite solution space. However there is no elliptic continuation analogous to $\partial \bar{\partial}$.

EXAMPLE 4'. Perturbing the symplectic form $\omega$ to a nondegenerate form $\widetilde{\omega}$ such that $d\left((\sim \widetilde{\omega})^{-1} d \widetilde{\omega}\right) \neq 0$, the elliptic complex

$$
\Lambda^{0} \stackrel{d}{\rightarrow} \Lambda^{1} \stackrel{\pi d}{\rightarrow} \Lambda^{2} / \tilde{\omega}
$$

is locally exact, but not formally exact. This is a quite general phenomenon which is not special to elliptic complexes.

\section{REFERENCES}

1. L. Ehrenpreiss, A fundamental principle for systems of linear differential equations with constant coefficients, and some of its applications, Proc. Internat. Sympos. Linear spaces (Jerusalem, 1960), Jerusalem Academic Press, Jerusalem; Pergamon, Oxford, 1961, pp. 161-174. MR 24 \#A3420.

2. H. Goldschmidt, Existence theorems for analytic linear partial differential equations, Ann. of Math. (2) 86 (1967), 246-270. MR 36 \#2933.

3. V. Guillemen and M. Kuranishi, Some algebraic results concerning involutive subspaces, Amer. J. Math. 90 (1968), 1307-1320. MR 39 \#2184.

4. M. Kuranishi, Involutive property of resolutions of differential operators, Nagoya Math. J. 27 (1966), 419-427. MR 37 \#943.

DEPARTMENT OF MATHEMATICS, COLUMBIA UNIVERSITY, NEW YORK, NEW YORK 10027 